\title{
Unusual conservative treatment of a complicated pacemaker pocket infection: a case report
}

Wanqiu Kang ${ }^{1}$, Xiaoming Chen ${ }^{2}$, Zicheng Li ${ }^{2}$, Aidong Zhang ${ }^{2 *}$, Jingwen Liu ${ }^{1}$, Liqiong Yu ${ }^{1}$ and Yingzhen Wen ${ }^{1}$

\begin{abstract}
Background: For patients with complicated generator pocket infection, expert consensuses universally advocate complete device and leads removal followed by delayed replacement on the contralateral side. We cured our patient by partial generator removal and reimplantation of sterilized pulse generator on the ipsilateral side. We also performed a literature review about incomplete removal therapy for the management of cardiac implantable electronic device infection.
\end{abstract}

Case presentation: An 86-year-old Chinese Han man was diagnosed as having third-degree atrioventricular block and received a permanent double-chamber pacemaker in his left prepectoral area 15 years ago. Nine years later, the entire system was removed because of confirmed infection, and a new device was reimplanted in the contralateral area. He developed skin necrosis around the pacemaker pocket after 1 year, and his generator was renewed without leads extraction at another medical center. He was subsequently admitted several times for surgical tissue debridement at another institution due to extended skin necrosis. At the time of the new admission, he had severe infection, heart failure, and hypoalbuminemia. He was diagnosed as having complicated pacemaker pocket infection. Our preferred treatment strategy was for complete removal of both the generator and transvenous pacing leads, and we intended to implant an epicardial pacemaker in our patient if necessary. However, he rejected the treatment strategy and firmly refused to replace his generator. We had to attempt a novel pacemaker-preserving strategy considering our patient's severe comorbidities. Finally, we cured him by partial generator removal and reimplantation of the sterilized pulse generator on the ipsilateral side. There was no sign of wound dehiscence or infection during a 6-month follow-up.

Conclusions: We would posit that partial removal of infected generators combined with conservative treatment may be a proper treatment of complicated generator pocket infection, especially for those who are susceptible to cardiac complications. Reimplantation of a sterilized pulse generator on the ipsilateral side may be an option if patients reject a new device and contralateral vascular condition is not really suitable. Opting for such treatment should be at the consideration of the primary care physician based on the condition of the patient.

Keywords: Complicated pacemaker pocket infection, Conservative treatment, Partial removal, Microbes, Antimicrobial therapy, Temporary pacing, Reimplantation

\footnotetext{
* Correspondence: zhangad1003@126.com

${ }^{2}$ Department of Cardiology, Guangzhou Overseas Chinese Hospital, The First

Affiliated Hospital of Jinan University, Guangzhou 510630, China

Full list of author information is available at the end of the article
}

(C) The Author(s). 2019 Open Access This article is distributed under the terms of the Creative Commons Attribution 4.0 International License (http://creativecommons.org/licenses/by/4.0/), which permits unrestricted use, distribution, and reproduction in any medium, provided you give appropriate credit to the original author(s) and the source, provide a link to the Creative Commons license, and indicate if changes were made. The Creative Commons Public Domain Dedication waiver (http://creativecommons.org/publicdomain/zero/1.0/) applies to the data made available in this article, unless otherwise stated. 


\section{Background}

An expansion in the clinical indications for implantation of cardiovascular implantable electronic devices (CIEDs), such as bradycardia, tachyarrhythmia, and heart failure, has led to a significant increase in the use of CIEDs over the past several decades $[1,2]$. Concurrently, CIED infection (CIEDI) has become increasingly prevalent [3]. Sandoe et al. defined complicated pacemaker pocket infection as pacemaker pocket infection with evidence of lead involvement, systemic symptoms of infection, or positive blood cultures [4]. For patients with complicated pacemaker pocket infection, expert consensuses universally advocate complete device and leads removal followed by delayed replacement on the contralateral side [4-7]. Unfortunately, some patients may not be candidates for device removal due to multiple comorbidities, limited life expectancy, or personal preference, which leads to reassessment of the optimal management strategies for these infections. We report a case of a patient with complicated pacemaker pocket infection who was cured by partial generator removal, reimplantation of the sterilized pulse generator on the ipsilateral side, debridement, and antimicrobial therapy. Few studies in the literature have reported such conservative treatment.

\section{Case presentation}

An 86-year-old Chinese Han man, with a known history of hypertension, heart failure, and chronic kidney disease, was diagnosed as having third-degree atrioventricular block and received a permanent double-chamber pacemaker in the left prepectoral area 15 years ago. Nine years later, the entire system (generator and leads) was removed because of confirmed infection, and a new device was reimplanted in the contralateral area. Unfortunately, he developed skin necrosis around the pacemaker pocket after 1 year and the generator was renewed without leads extraction at another medical center. After this procedure, a focal area at the mid portion of the wound failed to fully heal. He was subsequently admitted several times due to extended skin necrosis with massive purulent secretion and cellulitis around the incision site. His primary physician used multiple courses of antibiotics, local wound care, and debridement. This conservative management was continued for 5 years at another institution. There was ongoing pressure necrosis of the overlying skin which led to the gradual extrusion of his leads.

No social, environmental, family, or employment histories were related to his illness. He was born in China and has been living in Guangzhou for nearly 60 years. There is no hereditary disease in his family. He has a son who is in good health. He was an engineer before he retired 26 years ago. The following orally administered medications were given regularly to control his hypertension, heart failure, and chronic kidney disease in other hospitalizations: benazepril (10 $\mathrm{mg}$ once daily), niaoduqing (Chinese herbal medicine) particles $(5 \mathrm{~g}$ three times daily), furosemide ( $20 \mathrm{mg}$ once daily), and spironolactone $(20 \mathrm{mg}$ once daily). Throughout his periods of infection in other hospitals, his doctors once treated him with intravenously administered levofloxacin $(500 \mathrm{mg}$ once daily)/ciprofloxacin ( $200 \mathrm{mg}$ every 12 hours)/Tazocin (piperacillin-tazobactam; $4.5 \mathrm{~g}$ every 8 hours)/latamoxef ( $2 \mathrm{~g}$ twice daily)/ceftriaxone ( $\mathrm{g}$ once daily).

At the time of the new admission to our hospital, he was looking chronically ill. He was febrile with a temperature of $38.0^{\circ} \mathrm{C}$, and felt short of breath (New York Heart Association Functional Classification III). Oxygen saturation was $90-95 \%$ on room air. He was hemodynamically stable with a blood pressure of 165/74 $\mathrm{mmHg}$, and heart rate of 63 beats per minute. He has smoked tobacco for more than 50 years and never drinks alcohol. A physical examination revealed adherence of skin to the device with overt erosion and draining sinus could be observed on the right side of his upper chest (Fig. 1). We could see pus when squeezing the surgical incision. A cardiovascular examination was unremarkable. No evidence of infective endocarditis was observed. A chest examination showed bilateral basal crepitations. Severe edema was found in his penis, scrotum, and lower extremities. Laboratory test values are summarized in Table 1. A transthoracic echocardiography (TTE) displayed that his left ventricular ejection fraction was $52 \%$, and the result showed no evidence of vegetation attached to heart valves. Because of poor quality of TTE for diagnosis of infective endocarditis, a transesophageal

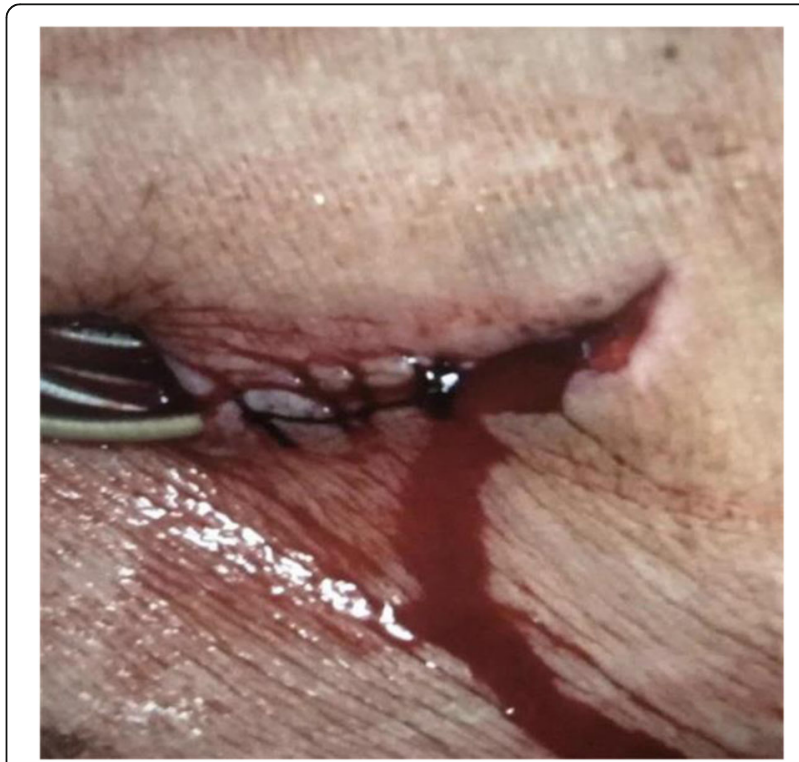

Fig. 1 Appearance of the wound at the time of admission. Adherence of skin to the leads was eroded overtly and draining sinus can be observed on the right side of the upper chest 
Table 1 Laboratory test results on admission

\begin{tabular}{lll}
\hline Test & Patient's value & Normal range \\
\hline WBC & $8.10 \times 10^{9} / \mathrm{L}$ & $4-10$ \\
NEUT\% & $79.60 \%$ & $50-70$ \\
PCT & $0.67 \mathrm{ng} / \mathrm{ml}$ & $0-0.1$ \\
CRP & $14.05 \mathrm{mg} / \mathrm{L}$ & Inflammation $>10.0$ \\
Urea & $12.48 \mathrm{mmol} / \mathrm{L}$ & $1.5-7.5$ \\
Creatinine & $170 \mathrm{umol} / \mathrm{L}$ & $32-106$ \\
e-GFR & $30 \mathrm{~mL} / \mathrm{minute}$ per $1.73 \mathrm{~m}^{2}$ & $>60$ \\
UA & $539 \mathrm{umol} / \mathrm{L}$ & $210-430$ \\
ALT & $10 \mathrm{U} / \mathrm{L}$ & $9-50$ \\
AST & $22 \mathrm{U} / \mathrm{L}$ & $15-40$ \\
Albumin & $25 \mathrm{~g} / \mathrm{L}$ & $35-52$ \\
TCHOL & $2.6 \mathrm{mmol} / \mathrm{L}$ & $3.1-5.7$ \\
LDL_C & $1.42 \mathrm{mmol} / \mathrm{L}$ & $1.57-3.76$ \\
D-Dimer & $2350 \mathrm{microg} / \mathrm{L}$ & $80-500$ \\
Troponin I & $<0.010 \mathrm{microg} / \mathrm{L}$ & $0.01-0.023$ \\
CK & $47 \mathrm{U} / \mathrm{L}$ & $26-174$ \\
NT-proBNP & $4150 \mathrm{ng} / \mathrm{L}$ & $300-900$ \\
\hline ALTani aming &
\end{tabular}

$A L T$ alanine aminotransferase, AST aspartate aminotransferase, $C K$ creatine kinase, CRP C-reactive protein, e-GFR estimated glomerular filtration rate, $L D L_{-} C$ low density lipoprotein cholesterol, NEUT\% the percentage of neutrophil granulocyte, NT-proBNP N-terminal pro-brain natriuretic peptide, $P C T$ procalcitonin, TCHOL total cholesterol, UA uric acid, WBC white blood cell count

echocardiography (TEE) was requested. However, our patient refused the examination firmly. Therefore, vegetation associated with device leads and endocardium could not be completely eliminated.

Blood and pocket secretions of our patient were sampled and cultured for aerobic, anaerobic bacteria and fungi respectively. After this procedure, he started receiving an intravenously administered broad-spectrum antibiotic (moxifloxacin $400 \mathrm{mg}$ once daily). Simultaneously, the wound was irrigated thoroughly with chlorhexidine, hydrogen peroxide, and saline every day (Fig. 2). On hospital day 7, the result of the blood culture revealed Staphylococcus epidermidis, and Corynebacterium striatum grew in the pus excretion culture. The pus Gram stain demonstrated Gram-positive, non-spore, rod-shaped bacteria, short but straight, which were arranged irregularly. No other strain was found in the Gram stain. The S. epidermidis was highly sensitive to penicillin, gentamicin, tetracycline, erythromycin, linezolid, vancomycin, and tigecycline; the S. epidermidis was moderately sensitive to levofloxacin, ciprofloxacin, and moxifloxacin, but not sensitive to cephalosporins. The $C$. striatum was highly sensitive to penicillin, cefoxitin, erythromycin, gentamicin, vancomycin, and teicoplanin; the C. striatum was moderately sensitive to clindamycin, but not sensitive to levofloxacin. Two sets of subsequent blood and secretion cultures after antibiotic therapy had confirmed the result. He was diagnosed as having complicated pacemaker pocket infection.

The intravenously administered antibiotic was changed to penicillin (3,200,000 IU every 8 hours) according to the antimicrobial drug susceptibility profile of our patient. Considering that he had severe infection, heart failure, and hypoalbuminemia, we treated him with intravenously administered immunoglobulin ( $2.5 \mathrm{~g}$ once daily), human albumin ( $10 \mathrm{~g}$ once daily), and furosemide $(20 \mathrm{mg}$ once daily). At the same time, fosinopril sodium (10 mg once a day), furosemide ( $20 \mathrm{mg}$ twice daily), and spironolactone (20 mg twice daily) were taken orally to control hypertension and reduce severity of heart failure. Orally administered niaoduqing (Chinese herbal medicine) particles ( $5 \mathrm{~g}$ three times daily) were also taken to improve renal function. After 1 month of conservative treatment, he was afebrile and his heavy breathing had improved.

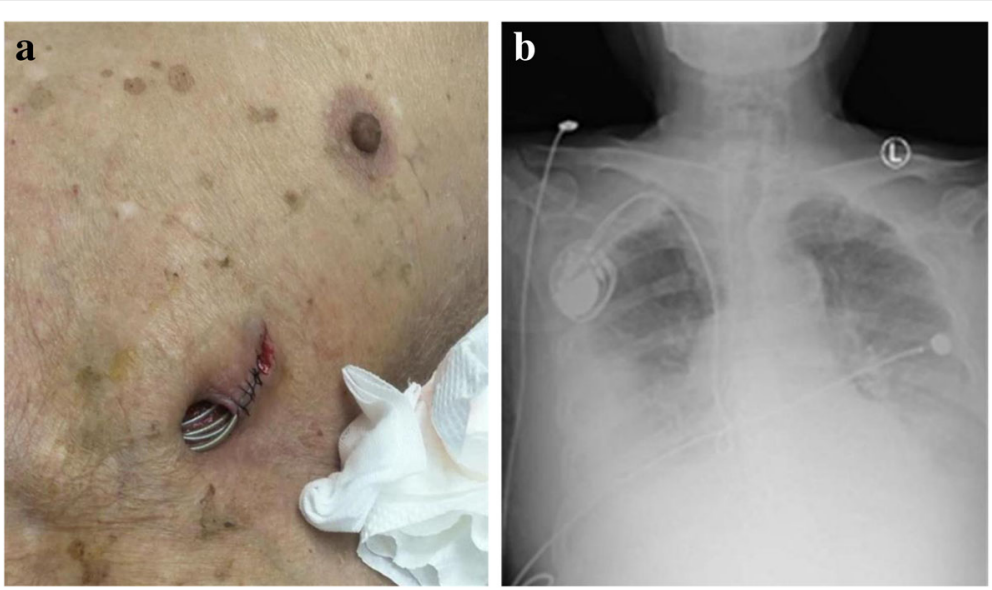

Fig. 2 a Appearance of the wound after irrigation (second week in the hospital). b Preoperative chest X-ray showed the generator was in the infraclavicular region 
The Holter monitor and pacemaker program suggested that he was not completely dependent on the pacemaker. Our preferred treatment strategy was for complete removal of both the generator and transvenous pacing leads, and we intended to implant an epicardial pacemaker in our patient if necessary. However, he rejected the treatment strategy, and refused to replace his generator because of economic factors. We had to attempt a novel device-preserving strategy considering our patient's severe comorbidities. Subsequently, the generator was extracted and immersed in povidone-iodine for sterilization. The lead was disconnected from the generator, capped, and allowed to remain in situ. Because our patient was not completely dependent on the pacemaker, a temporary pacemaker was not used. His heart rate fluctuated from 33 to 68 times per minute after extracting pulse generator. Necrotic tissue was extensively debrided to shorten the time of wound healing. During this time, intravenously administered antibiotics and cardiac monitoring were continued. After 10 days, when blood culture was negative, the sterilized generator was successfully reimplanted in a different position on the same side (Fig. 3). The generator remained out of the pocket for 10 days in total. The surgical wound healed rapidly. Seven days after device reimplantation, he was discharged with an extensive list of medications: $10 \mathrm{mg}$ of fosinopril sodium once daily; $20 \mathrm{mg}$ of furosemide once daily; $20 \mathrm{mg}$ of spironolactone once daily; $20 \mathrm{mg}$ of trimetazidine dihydrochloride three times daily; $5 \mathrm{~g}$ of niaoduqing (Chinese herbal medicine) particles three times daily; and $150 \mathrm{mg}$ of iron polysaccharide complex capsules once daily. No orally administered antibiotic was used after discharge.

He showed up to his follow-up appointments every month. There was no sign of wound dehiscence (Fig. 4) and the pacemaker worked properly during a 6-month follow-up. Clinical markers of infection were normal and recorded (Fig. 5). At the time of his last follow-up appointment, he was afebrile with a temperature of $37.2{ }^{\circ} \mathrm{C}$. Oxygen saturation was $100 \%$ on room air. His blood pressure was $150 / 61 \mathrm{mmHg}$, and heart rate was 52 beats per minute. A physical examination revealed there were surgical scars but no sign of wound dehiscence on the right side of his upper chest. There was still mild edema in lower extremities, but he did not have obvious shortness of breath at rest. A chest examination showed no bilateral basal crepitations. A cardiovascular examination was unremarkable. No heart murmur could be heard. A neurological examination revealed that his functions of sensation and movement were normal, and he was able to carry out daily activities independently. A timeline to show disease progression is shown in Fig. 6.

\section{Discussion}

We presented a patient with complicated generator pocket infection. Leads removal might not have been an option for him because he was at very high risk because of age and concomitant diseases. We treated him with partial generator removal and reimplantation of the sterilized pulse generator on the ipsilateral side. Few studies in the literature have reported such therapeutic strategy.

CIEDI is a serious cardiovascular disease and it is associated with a high mortality. In a large cohort of patients with CIEDI, the 30 -day mortality rate was $5.5 \%$, and 1-year mortality was $14.6 \%$ [8]. Erosion of any part of the CIED indicates contamination of the entire system, and complete device removal should be performed. Conservative antibiotic therapy combined with limited debridement and irrigation of infected sites without removal of the infected device system may lead to poor outcomes. Le et al. reported that antimicrobial therapy without device removal was associated with a sevenfold increase in 30-day mortality [8].

Although complete removal of an infected CIED is first-line therapy, there may be complications about device removal. According to previous statistics, the risk

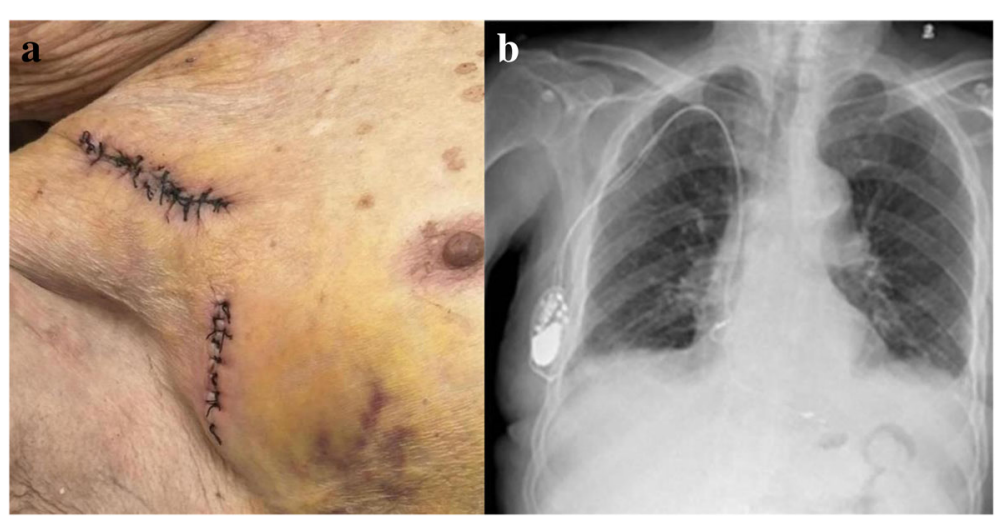

Fig. 3 a Appearance of the wound after reimplantation. $\mathbf{b}$ Postoperative chest X-ray showed the generator was reimplanted in different position on the same side 


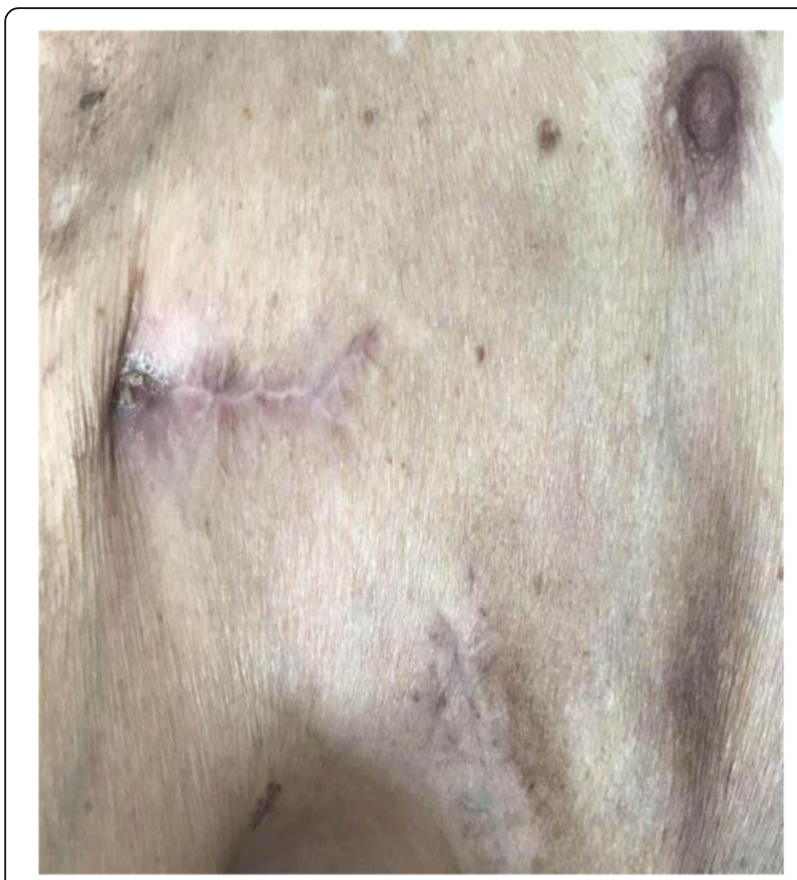

Fig. 4 Complete healing of the wound (sixth month after discharge)

of major complications (for example, vascular laceration, death) approaches $2 \%$ for all extraction attempts [6]. Risk factors associated with procedural complications and death are not completely known. In the LExICon study [9], patients with a body mass index (BMI) $<25$ $\mathrm{kg} / \mathrm{m}^{2}$ were more likely to experience major adverse events related to the lead extraction procedure. Some risk factors such as low BMI, renal disease, diabetes mellitus, and extraction for infection, increased the risk for death during their hospitalization. Another study confirmed the relevance of these risk factors. Brunner et al. produced a nomogram for the risk of 30-day all-cause death after leads extraction [10]. The factors with the highest predictive value for death were heart failure, older age, abnormal BMI, and extraction for infection [10].

The patient we described was elderly, and suffered from heart failure (New York Heart Association Functional Class III), chronic renal failure, and low BMI $\left(18 \mathrm{~kg} / \mathrm{m}^{2}\right)$. The removal of the entire pacing system could predispose our patient to unexpected events. In addition, he preferred conservative therapy as he had experienced complete extraction of the pulse generator and leads on the contralateral side 6 years previously. As a result, we decided to partially remove the generator combined with conservative treatment (antibiotic therapy, debridement, and irrigation).

Some case reports and small case series suggested that salvage of an infected CIED may sometimes be successful. Lopez saved the pulse generators and leads of five patients by using mechanical means (scrubbing and pulsed lavage) and a closed antimicrobial irrigation system [11]. Tan et al. retrospectively analyzed 33 patients who initiated chronic antibiotic suppression without device removal, only $18 \%$ (6/33) developing into relapse within 1 year [12]. Peacock et al. reported on 127 patients for whom conservative management with device retention was attempted, $20 \%$ met the study definition for successful salvage [13]. Even with more serious forms of device infection, such as leads endocarditis, medical management without device removal may be successful. In the study of Tascini and colleagues [14], two out of nine patients with CIED endocarditis were too sick for the removal of their CIED, and were cured with $6 \mathrm{mg} / \mathrm{kg}$ of daptomycin without adverse event.

There have been some reports concerning the partial device removal of infected CIED [15-17]. Table 2 was used to compare our case with cases that were available in the literature. In those studies, incomplete removal resulted in greater rates of infection relapse. According to those studies, we may successfully manage the patient with conservative treatment and partial removal of device, but the likelihood of infection relapse in our patient was considerable. We should have a longer follow-up.

It is sometimes difficult to determine the causative organism. This is because the results of each culture from different sites may suggest different organisms. Bongiorni et al. reported on one of the largest (1204 patients) microbiology studies in CIEDI [18]. They investigated 116 cases of materials from pockets and 359 cases where blood samples were obtained for culture. The results were consistent with those from electrodes in $59 \%$ and $35 \%$ of cases respectively. Golzio et al. gave a definition of causative organism as consistent species detected from at least two different sites [19]. Each blood culture was counted as a different material, and pocket material was considered a single site. Nine sets of blood and pocket excretion samples of our patient were cultured for aerobic, anaerobic bacteria and fungi. Three blood cultures revealed S. epidermidis, four revealed other coagulase-negative Staphylococcus, and the other two were negative. Four pocket excretion cultures revealed $C$. striatum and the other five were negative. Therefore, we can regard coagulase-negative Staphylococcus as the causative organism. According to the study findings of Fukunaga et al., the causative organism of the CIEDI was mainly Staphylococcus aureus and coagulase-negative Staphylococci (for example, S. epidermidis), but $S$. aureus showed a higher concordance in leads and pocket cultures than coagulase-negative Staphylococci [20]. Gram-positive bacteria (excluding Staphylococcus), such as Corynebacterium species, showed relatively low concordance, which meant a benign coexisting organism [20]. In the case of our patient, blood cultures were coagulase-negative Staphylococcus 


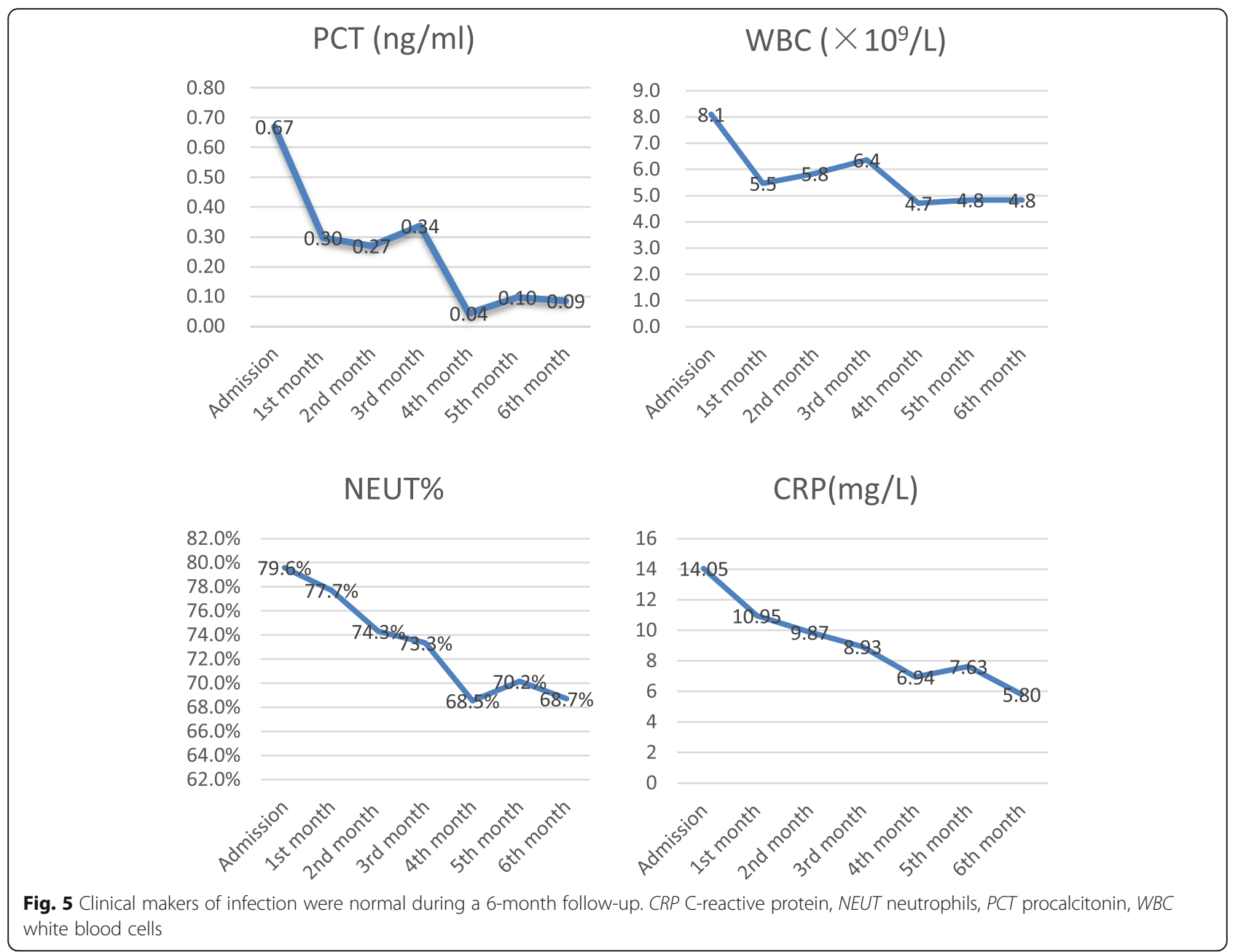

but C. striatum grew in the pocket excretion cultures. This could be explained by the use of antibiotic therapy previously and preexisting surgical tissue debridement before his new admission. Eventually, we identified coagulasenegative Staphylococcus as the causative organism while $C$. striatum tended to coexist as a benign organism. As with most infections, an antimicrobial drug initially should cover common organisms broadly and antibiotic administration should begin after collection of blood and excretion cultures. Narrowing of the antimicrobial spectrum should be based on antimicrobial drug susceptibility. The duration of antibiotic treatment after removal of an infected device

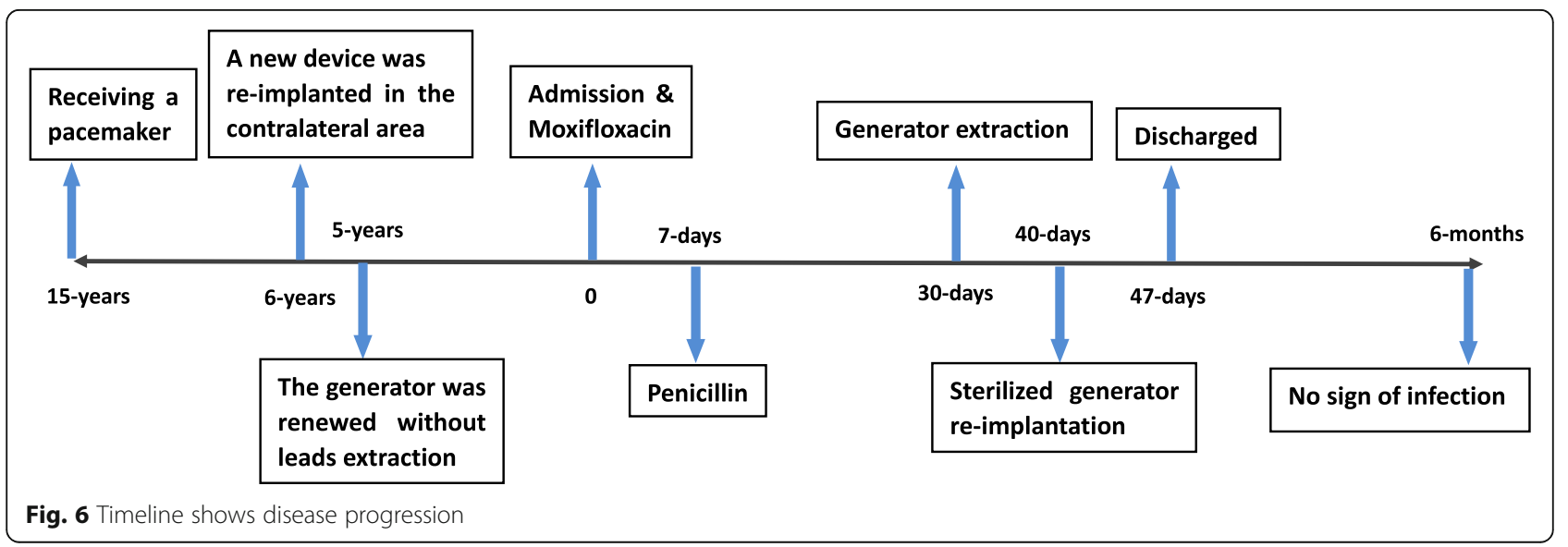


Table 2 Review of literature regarding salvage of cardiovascular implantable electronic devices

\begin{tabular}{|c|c|c|c|c|c|}
\hline Study & Infection, $n$ & Management, $n$ & Complications, $n$ & Success & $\begin{array}{l}\text { Follow-up } \\
\text { (months) }\end{array}$ \\
\hline $\begin{array}{l}\text { Present study, } \\
2018\end{array}$ & CIEDI (1) & $\begin{array}{l}\text { Partial removal + conservative therapy }+ \\
\text { sterilized generator reimplantation (1) }\end{array}$ & 0 & $100 \%$ & 6 \\
\hline $\begin{array}{l}\text { Lopez [11], } \\
2013\end{array}$ & CIEDI (5) & $\begin{array}{l}\text { Scrubbing }+ \text { pulsed lavage }+ \text { closed } \\
\text { antimicrobial irrigation system (5) }\end{array}$ & 0 & $100 \%$ & 19.2 \\
\hline $\begin{array}{l}\text { Tan et al. [12], } \\
2017\end{array}$ & CIEDI (33) & Chronic antibiotic suppression (33) & Infection relapse (6) & $82 \%$ & 12 \\
\hline $\begin{array}{l}\text { Peacock et al. } \\
{[13], 2018}\end{array}$ & CIEDI (127) & Device retention + antibiotics (127) & $\begin{array}{l}\text { Early failure of salvage (74); infection relapse (6); } \\
\text { chronic suppression (7); death (14) }\end{array}$ & $20 \%$ & 6 \\
\hline $\begin{array}{l}\text { Tascini et al. } \\
{[14], 2012}\end{array}$ & $\begin{array}{l}\text { CIED } \\
\text { endocarditis (2) }\end{array}$ & Daptomycin (2) & 0 & $100 \%$ & 17 \\
\hline $\begin{array}{l}\text { Margey et al. } \\
{[15], 2010}\end{array}$ & CIEDI (13) & $\begin{array}{l}\text { Partial removal or conservative } \\
\text { therapy (13) }\end{array}$ & Infection relapse (8); death (1) & $25 \%$ & 36 \\
\hline
\end{tabular}

CIED cardiovascular implantable electronic device, CIEDI cardiovascular implantable electronic device infection

varied in different studies. In general, the more residual devices left in place, the longer the duration of treatment. We used antimicrobial therapy for 47 days in total and for 7 days after reimplantation in our patient, which was in accordance with the guidelines [4].

The pulse generator of our patient was removed for sterilization. For patients who are pacemaker-dependent, temporary pacing is required as a bridge to the reimplantation of a new permanent device [7]. However, it has been associated with higher mortality [21], and increased risk of infection [22]. We daringly did not use a temporary pacemaker in our patient, although his heart rate was sometimes only 33 times per minute. Customary treatment for lead infection would involve contralateral implantation of a new device. Given complete contralateral venous occlusion and our patient's rejection of a new generator, the sterilized prior generator was reimplanted in a different position on the same side connecting old electrodes. No report has introduced the surgical technique to date.

\section{Conclusions}

We would posit that partial removal of infected generators combined with conservative treatment may be a proper treatment of complicated generator pocket infection, especially for those who are susceptible to cardiac complications or lack the necessary financial resources. Although not a widely accepted practice, reimplantation of the sterilized pulse generator on the ipsilateral side may be an option if a patient rejects a new device and contralateral vascular condition is not really suitable. Opting for such treatment should be at the consideration of the primary care physician based on the condition of the patient, and the pulse generator and remaining leads must be completely sterilized to eradicate infection. On the basis of a limited number of patients, further studies are needed to better define the optimal subpopulation that would benefit from the approach.

\section{Abbreviations}

BMI: Body mass index; CIED: Cardiovascular implantable electronic device: CIEDI: Cardiovascular implantable electronic device infection; $C$. striatum: Corynebacterium striatum; S. aureus: Staphylococcus aureus; S. epidermidis: Staphylococcus epidermidis; TTE: Transthoracic echocardiography

\section{Acknowledgements}

Not applicable.

Funding

This work was not supported by any grants.

Availability of data and materials

All data used during the present study are available from the corresponding author on reasonable request.

\section{Authors' contributions}

$W K, J$, and $L Y$ prepared the clinical information and wrote the manuscript. $A Z$ and $Y W$ reviewed the manuscript. $Z L$ and $X C$ revised the article in detail. All authors read and approved the final manuscript.

Ethics approval and consent to participate

This case report was ethically approved and consented by the Ethics Committee of Guangzhou Overseas Chinese Hospital Affiliated to Jinan University.

\section{Consent for publication}

Written informed consent was obtained from the patient for the publication of this case report and any accompanying images. A copy of the written consent is available for review by the Editor-in-Chief of this journal.

\section{Competing interests}

The authors declare that they have no competing interests.

\section{Publisher's Note}

Springer Nature remains neutral with regard to jurisdictional claims in published maps and institutional affiliations.

\section{Author details}

${ }^{1}$ The First Clinical Medical College of Jinan University, Guangzhou 510630, China. ${ }^{2}$ Department of Cardiology, Guangzhou Overseas Chinese Hospital, The First Affiliated Hospital of Jinan University, Guangzhou 510630, China. 
Received: 18 August 2018 Accepted: 18 January 2019

Published online: 03 March 2019

\section{References}

1. Kurtz SM, Ochoa JA, Lau E, et al. Implantation trends and patient profiles for pacemakers and implantable cardioverter defibrillators in the United States: 1993-2006. Pacing Clin Electrophysiol. 2010;33(6):705-11.

2. Uslan DZ, Tleyjeh IM, Baddour LM, et al. Temporal trends in permanent pacemaker implantation: a population-based study. Am Heart J. 2008:155(5):896-903.

3. Sridhar AR, Lavu M, Yarlagadda V, et al. Cardiac implantable electronic device-related infection and extraction trends in the U.S. Pacing Clin Electrophysiol. 2017;40(3):286-93.

4. Sandoe JA, Barlow G, Chambers JB, et al. Guidelines for the diagnosis, prevention and management of implantable cardiac electronic device infection. Report of a joint working party project on behalf of the British Society for Antimicrobial Chemotherapy (BSAC, host organization), British Heart Rhythm Society (BHRS), British Cardiovascular Society (BCS), British Heart Valve Society (BHVS) and British Society for Echocardiography (BSE). J Antimicrob Chemother. 2015;70(2):334-59.

5. Baddour LM, Epstein AE, Erickson CC, et al. Update on cardiovascular implantable electronic device infections and their management: a scientific statement from the American Heart Association. Circulation. 2010:121(3):458-77.

6. Wilkoff BL, Love CJ, Byrd CL, et al. Transvenous lead extraction: Heart Rhythm Society expert consensus on facilities, training, indications, and patient management: this document was endorsed by the American Heart Association (AHA). Heart Rhythm. 2009;6(7):1085-104.

7. Kusumoto FM, Schoenfeld MH, Wilkoff BL, et al. 2017 HRS expert consensus statement on cardiovascular implantable electronic device lead management and extraction. Heart Rhythm. 2017;14(12):e503-51.

8. Le KY, Sohail MR, Friedman PA, et al. Impact of timing of device removal on mortality in patients with cardiovascular implantable electronic device infections. Heart Rhythm. 2011;8(11):1678-85.

9. Wazni O, Epstein LM, Carrillo RG, et al. Lead extraction in the contemporary setting: the LExICon study: an observational retrospective study of consecutive laser lead extractions. J Am Coll Cardiol. 2010;55(6):579-86.

10. Brunner MP, Yu C, Hussein AA, et al. Nomogram for predicting 30-day all-cause mortality after transvenous pacemaker and defibrillator lead extraction. Heart Rhythm. 2015;12(12):2381-6.

11. Lopez JA. Conservative management of infected pacemaker and implantable defibrillator sites with a closed antimicrobial irrigation system. Europace. 2013;15(4):541-5.

12. Tan EM, Desimone DC, Sohail MR, et al. Outcomes in patients with cardiovascular implantable electronic device infection managed with chronic antibiotic suppression. Clin Infect Dis. 2017:64(11):1516-21.

13. Peacock JE Jr, Stafford JM, Le K, et al. Attempted salvage of infected cardiovascular implantable electronic devices: Are there clinical factors that predict success? Pacing Clin Electrophysiol. 2018;41(5):524-31.

14. Tascini C, Bongiorni MG, Di Cori A, et al. Cardiovascular implantable electronic device endocarditis treated with daptomycin with or without transvenous removal. Heart Lung. 2012;41(6):e24-30.

15. Margey R, McCann H, Blake G, et al. Contemporary management of and outcomes from cardiac device related infections. Europace. 2010;12(1):64-70

16. Pichlmaier M, Knigina L, Kutschka I, et al. Complete removal as a routine treatment for any cardiovascular implantable electronic device-associated infection. J Thorac Cardiovasc Surg. 2011;142(6):1482-90.

17. Goya M, Nagashima M, Hiroshima K, et al. Lead extractions in patients with cardiac implantable electronic device infections: Single center experience. J Arrhythm. 2016;32(4):308-12.

18. Bongiorni MG, Tascini C, Tagliaferri E, et al. Microbiology of cardiac implantable electronic device infections. Europace. 2012;14(9):1334-9.

19. Golzio PG, Vinci $M$, Anselmino $M$, et al. Accuracy of swabs, tissue specimens, and lead samples in diagnosis of cardiac rhythm management device infections. Pacing Clin Electrophysiol. 2009; 32(suppl 1):S76-80

20. Fukunaga $M$, Goya $M$, Nagashima $M$, et al. Identification of causative organism in cardiac implantable electronic device infections. J Cardiol. 2017;70(5):411-5.
21. Deharo JC, Quatre A, Mancini J, et al. Long-term outcomes following infection of cardiac implantable electronic devices: a prospective matched cohort study. Heart. 2012;98(9):724-31.

22. Klug D, Balde M, Pavin D, et al. Risk factors related to infections of implanted pacemakers and cardioverter-defibrillators: results of a large prospective study. Circulation. 2007;1 16(12):1349-55.

\section{Ready to submit your research? Choose BMC and benefit from:}

- fast, convenient online submission

- thorough peer review by experienced researchers in your field

- rapid publication on acceptance

- support for research data, including large and complex data types

- gold Open Access which fosters wider collaboration and increased citations

- maximum visibility for your research: over $100 \mathrm{M}$ website views per year

At BMC, research is always in progress.

Learn more biomedcentral.com/submissions 